\title{
TOWARDS A CYBERSECURITY CULTURE TOOL THROUGH A HOLISTIC, MULTI-DIMENSIONAL ASSESSMENT FRAMEWORK
}

\author{
Kanaris Bounas, Anna Georgiadou, Michalis Kontoulis, Spiros Mouzakitis and Dimitrios Askounis \\ National Technical University of Athens \\ Iroon Politexniou 9, Zografou, Greece
}

\begin{abstract}
Cyber-security culture forms one of the best lines of defence against many cyber-attacks making it an ideal and efficient target to address, in order to better secure organisations. It has its foundations on the information security policies and procedures enforced by its management. It is also dictated and formed by the behaviour, perceptions and attitudes of its employees. These will form the basis of our model which aims to quantify these theoretical constructs in order to analyse them and provide feedback. The close examination of existing security assessment tools and frameworks while identifying the most important security components and sections which need to be addressed and evaluated, resulted in our proposition of a state-of-the-art cyber-security culture tool. The presented approach aims to address existing gaps by adopting a holistic perspective that incorporates both organisational and individual aspects of security culture, including behavioural traits that affect and risk the security level of the company. Our approach and tool will be applied to the EPES sector, where the timely assessment of the security culture is considered essential and of high impact.
\end{abstract}

\section{KEYWORDS}

Cybersecurity Culture, Assessment, Awareness, Security Behaviour

\section{INTRODUCTION}

Due to the radical technological development of the last decades, cybersecurity has emerged into one of the most demanding and troubling scientific areas absorbing large amount of time, effort, research and funds. Many attempts have been made in the past to define, implement and establish information security frameworks applicable to any kind of organization regardless of structure, size or industry. Although unsuccessful in concluding to a holistic model, they managed to prove the significance of the human element in information security while acknowledging the need for a more sophisticated and generic approach nowadays described under the term "information security culture".

Many definitions have been introduced in the past, focusing on various aspects of the information security culture. Although, researchers agree on the levels and the objectives, they tend to differentiate on the domains and components they recognize and try to assess while evaluating the information security culture of an organization.

One of the most important parts of information security is the information security assessment that should be conducted in order to identify possible weaknesses in the organization. This is no different when studying socio-cultural behavior in information security. A complete assessment will provide all the weaknesses that arise in the organization due to either its employees or its policies and procedures that it has established. After identifying them, finding solutions and improving security as a whole becomes much easier and cost efficient.

This paper focuses on finding an appropriate way to conduct an assessment that will provide results that cover every aspect of socio-cultural behaviour in information security. It will also elaborate the most commonly used standards and frameworks on this area while identifying their deficiencies and lacks which, consequently, lead to the need of seeking new methodologies and approaches. A set of concepts and the respective features needed, lead to the design and implementation of a cyber-security culture tool targeting some of the most demanding issues of this field. 


\section{BACKGROUND}

Information security components can be described as the principles that enable the implementation and maintenance of information security while cultivating an appropriate level of information security culture and minimizing risks posed to information assets (Da Veiga \& Eloff, 2007). Although their significance is commonly accepted, they have been approached with many ways by the various information security culture frameworks over the last century.

In 1995, BSI Group published BS 7799 as a standard, which consisted of several parts. The first part was revised in 1998 and contained the best practices for information security management. In 2000, it was adopted by ISO as ISO/IEC 17799:2000 "Information technology - Code of practice for information security management". It was then later revised in 2005 as ISO/IEC 17799:2005 and finally incorporated in the ISO 27000 series of standards as ISO/IEC 27002:2007 in July 2007. The later revision of this standard was in 2013.

The second part of BS 7799 was published by BSI in 1999, known as BS 7799 Part 2 and titled "Information Security Management Systems - Specification with guidance for use." BS 7799-2 focused on how to implement an Information security management system (ISMS). In November 2005, this part was adopted by ISO as ISO/IEC 27001:2005 "Information technology — Security techniques - Information security management systems - Requirements". The later revision of this standard was in 2013.

In 2007, the Information Security Governance framework is proposed as a starting point for an organization to govern information security by developing guidelines and implementing controls to address risks identified by the organizations (Da Veiga \& Eloff, 2007). It is partitioned into four levels trying to combine the pros of all its predecessors while providing a single point of reference for the governance of information security to inculcate an acceptable level of information security culture.

In 2017, ENISA published the CSC Framework according to which, the concept of Cybersecurity Culture (CSC) refers to the knowledge, beliefs, perceptions, attitudes, assumptions, norms and values of people regarding cybersecurity and how they manifest themselves in people's behaviour with information technologies (ENISA, 2017). Obeying to its principles and guidelines, the Security CLTRe Toolkit breaks CSC into seven dimensions, measured by a staff questionnaire, leading to a graphical representation of the organization's security culture score (Petric \& Roer, 2018).

\section{METHODOLOGY}

\subsection{Model Definition}

Having glanced into the information security culture background and having identified the most commonly accepted frameworks and tools, we proceeded in conducting a thorough literature review and detailed research on the elements that constitute the critical factors influencing and finally formulating the cyber-security culture.

Much research has been conducted over the years with an apparent trend to move from a technical approach, towards information security, to a socio-cultural approach (Da Veiga, Martins, \& Eloff, 2007; ENISA, 2018). In other words, techniques such as technical analyses and testing of information systems, mathematical models or statistical computations of risks and economic analyses of investment justification tend to be substituted by behavioural, organizational and criminological theories (Bernik \& Prislan, 2016) as the basis of security assessment. Our tool aims to bridge the apparent gap between these two fundamentally different approaches by combining their methods and enhancing their numerous advantages.

We defined a theoretical model to portray the most important cyber-security domains and sub-domains which need to be evaluated and properly assessed in order to integrate the overall culture at an organizational level. This model aims in depicting the key metrics to be measured along with their dependencies, influences and varieties and focuses on a holistic approach that can be applied to any type of organization. When all those metrics are identified, the model will gather all the results from the assessment conducted and use them to illustrate a uniform representation of the everyday reality of an organization, as well as map the weaknesses identified in the assessment with several known cybersecurity threats. Recommendations for improvement will also be a function that the tool will provide. 
Initially, the tool will need to conduct the assessment in order for its other functions to be able to provide accurate outputs. An assessment cannot be conducted before first identifying all the necessary measurements that need to be taken into account. For this purpose, two different kind of measurements will be used. One category will assess the organization's policies and procedures, and the other will assess employees' behavior in relevance with information security. There will be several methods with which the assessments will be conducted, like questionnaires and tests, audits, etc. The assessments will be conducted in various waves, called assessment campaigns, and these will be repeated after a certain period of time.

In the next step, the assessment will provide a score to all the entities in the organization, ranging from each employee separately, to each department and finally the organization as a whole. Each entity will be scored in every relevant domain and sub-domain that describes the organization's information security culture. Various variables will be used to give a more accurate scoring, which will be customizable for each different organization. The results of the assessments will be available to only authorized users and will be protected from unauthorized access.

After the assessment's conclusion, the tool will identify all the known cybersecurity threats that are relevant to the organization. Then, it will map all the weaknesses detected in the assessment with the cybersecurity threats and evaluate which of them have high risk potential.

Finally, it will provide some guidelines, both on personal as well as on organizational level, which, if followed, will improve the organization's information security culture. For this purpose, the tool will create a personal profile for each employee, in which all the areas of information security that need to be improved will be highlighted, and possible actions for this improvement will be provided.

\subsection{Metric Quantification \& Tool Development}

Culture is constituted by collective values, norms, knowledge and behaviour which, as theoretical constructs and mistily notions, are characteristically hard to measure. Consequently, most of the scientific research focuses on its indirect measurement via its artefacts: management, work processes, procedures, policies, etc. (Schlienger \& Teufel, 2003). Thus, our challenge is the way of quantifying and measuring all the indicators defined by the previous steps while exploiting other sciences apart from information engineering.

The multidimensional model defined in our study demands an equally multidimensional implementation approach. Therefore, different evaluation and delivery methods, varying from the conventional ones, such as documentation analysis, physical and participative observation, narrative interviews, typical questionnaires (Schein, 1985; Despres, 1995), to the most radical, such as instructor-led, online, game, video and simulation based, shall be used (Abawajy, 2014).

First-level results gathered by these techniques need to be elaborated and finally transformed to value-added knowledge for both individuals and organizations leading to security cultural evolution. Data processing algorithms and advanced analytics shall be the foundations of our cyber-security culture tool. Even among business that are intrinsically interlinked with information systems not all members of the organisations have the same knowledge set and even when dealing with sector experts novel cyber visualisations have been observed to be either too complex or too basic for the intended users (Diane Staheli, 2014). As such it is necessary to ensure that our results are presented with the appropriate complexity and tools corresponding to the knowledge level of each user, from the personal to the organisational level, to achieve the maximum impact.

\section{APPLICATION}

Having finalized our tool, we shall proceed in applying our research and processes to different organizational types gathering information and calibrating our algorithms based on outputs, findings and user feedback. We will begin with implementing our tool in organizations belonging to the EPES sector, but our ultimate goal is to standardize our techniques and conclude to a cyber-security framework capable of assisting and boosting any type of business while becoming a valuable asset. The tool will be deployed into the servers of each organization, converting it into an internal tool of each organization. 


\section{CONSTRAINTS AND ISSUES}

While improving the cyber-security culture is a task of paramount importance there may be some hurdles that can impede our efforts which must be taken under consideration especially when applying the framework in a real-life environment.

Special attention, procedures and mechanisms need to be applied for the protection of personal data according to both the European Regulations (GDPR) as well as local government laws that apply to the organization using the toolset.

Lastly the main goal of the tool is to assess in a holistic manner the security culture of the organization. In-depth and hands-on technical security testing and vulnerability scanning are outside the scope of the tool and are expected to be used complimentary to our proposed tool.

\section{FUTURE WORK}

At a later stage, this model shall be enriched, tuned and tailored to the EPES (Electrical Power Energy Systems) value chain (generator, TSO, DSO, consumer) as part of a toolkit combining the latest technologies for vulnerability assessment (automated threat-modelling and security behaviour analysis), monitoring and protection (anomaly detection and DDoS mitigation) and learning and sharing (security information and event management) (Energy Shield, 2019). The toolkit will be validated in large-scale demonstrations by end-users while providing a unique level of security culture visibility.

\section{CONCLUSION}

In order to achieve an adequate information security level, you need to recognize the importance of the human factor in the security chain (Williams, 2009). Procedures and practices should focus on promoting a cyber-security culture which adapts and evolves as a living mechanism to the continuous demanding technological environment of this century (Wilson \& Hash, 2003). Therefore, a promising cyber-security culture tool needs to closely examine and monitor all the domains which formulate, directly or indirectly, the organizational reality while cultivating the employee engagement on a daily basis. Personalized recommendations, training programs, vulnerability identification and proactive initiatives are the ultimate goal of our multidimensional tool. To enhance its validity, after implementation completion, it shall need to be tested and proved against different business types and organizations while identifying possible deficiencies and proceeding in needed adjustments to satisfy all the originally set targets. Additional findings and results are expected during the pilot application of the tool operation.

\section{ACKNOWLEDGEMENT}

The cyber-security culture tool described in the previous paragraphs is being developed as part of the $\mathrm{H} 2020$ project, "Integrated Cybersecurity Solution for the Vulnerability Assessment, Monitoring and Protection of Critical Energy Infrastructures" (Energy Shield, 2019).

\section{REFERENCES}

Abawajy, J. (2014). User preference of cyber security awareness delivery methods. Behaviour \& Information Technology, 33(3), 237-248. doi:10.1080/0144929X.2012.708787

Bernik, I., \& Prislan, K. (2016). Measuring Information Security Performance with 10 by 10 Model for Holistic State Evaluation. (H. Song, Ed.) doi:10.1371/journal.pone.0163050 
Business and Advisory Committee to the OECD. (2004). Securing your business. An companion for small or entrepreneurial companies to the 2002 OECD Guidelines for the security of networks and information systems: Towards a culture of security. Paris: International Chamber of Commerce.

(n.d.). Cybersecurity Checklist Series. JMARK Business Solutions.

Da Veiga, A., \& Eloff, J. H. (2007). An Information Security Governance Framework. Information Systems Management, 24(4), 361-372. doi:10.1080/10580530701586136

Da Veiga, A., Martins, N., \& Eloff, J. H. (2007). Information security culture-validation of an assessment instrument. 11(1).

Despres, C.-N. (1995). Culture, Surveys, Culture Surveys and Other Obfuscations: A reply to Migliore and Martin. The Journal of Strategic Change, 4(2), 65-75.

Diane Staheli, T. Y. (2014). Visualization evaluation for cyber security: trends and future directions. VizSec '14, 49-56.

Eloff, J. H., \& Eloff, M. (2005). Information Security Architecture. Computer Fraud \& Security, 10-16. doi:10.1016/S1361-3723(05)70275-X

Energy Shield. (2019, 10 24). Retrieved from Energy Shield: https://energy-shield.eu/

ENISA. (2017). Cyber Security Culture in Organisations. European Union Agency For Network and Information Security. doi: $10.2824 / 10543$

ENISA. (2018). Cybersecurity Culture Guidelines: Behavioural Aspects of Cybersecurity. European Union Agency for Network and Information Security. doi:10.2824/324042

ISO/IEC 17799. (2005). Information technology - Security techniques - Code of practice for information security management. International Organization for Standardization.

ISO/IEC 27002. (2013). Information technology - Security techniques - Code of practice for information security controls. International Organization for Standardization.

Petric, G., \& Roer, K. (2018). To measure security culture: A scientific approach. CLTRe North America, Inc.

Schein, E. H. (1985). Organizational Culture and Lead-ership: A Dynamic View (1st ed.). San Francisco: Jossey-Bass.

Schlienger, T., \& Teufel, S. (2003). Analyzing Information Security Culture: Increasing Trust by an Appropriate Information Security Culture. 14th International Conference on Database and Expert Systems Applications (DEXA 2003). Prague: IEEE Computer Society.

Shetty N., S. G. (210). Competitive Cyber-Insurance and Internet Security. Economics of Information Security and Privacy, 229-247.

Williams, P. A. (2009). What Does Security Culture Look Like For Small Organizations? 7th Australian Information Security Management Conference. Perth: Edith Cowan University . doi:10.4225/75/57b4029530dea

Wilson, M., \& Hash, J. (2003). Building an Information Technology Security Awareness and Training Program. U.S. Department of Commerce. Washington: National Institute of Standards and Technology. Retrieved 10 10, 2019, from http://citadel-information.com/wp-content/uploads/2012/08/nist-sp800-50-building-information-security-awarenessprogram-2003.pdf

Zerlang, J. (2017). GDPR: a milestone in convergence for cyber-security and compliance. Network Security, Volume 2017, Issue 6, 8-11. 